\title{
Péter Pomozi : Kis nyelv - nagy stratégia. Az észt nyelvpolitikai modell
}

[Une petite langue, une grande stratégie. Le modèle estonien de politique linguistique], Budapest, 2011, 154 p.

\section{Eva Toulouze}

\section{(2) OpenEdition}

\section{Journals}

Édition électronique

URL : https://journals.openedition.org/efo/5546

DOI : $10.4000 /$ efo. 5546

ISSN : 2275-1947

Éditeur

INALCO

Édition imprimée

ISBN : 978-2-343-08571-5

ISSN : 0071-2051

Référence électronique

Eva Toulouze, «Péter Pomozi : Kis nyelv - nagy stratégia. Az észt nyelvpolitikai modell », Études finnoougriennes [En ligne], 47 | 2015, mis en ligne le 06 juillet 2016, consulté le 20 septembre 2021. URL http://journals.openedition.org/efo/5546 ; DOI : https://doi.org/10.4000/efo.5546

Ce document a été généré automatiquement le 20 septembre 2021.

Études finno-ougriennes est mis à disposition selon les termes de la Licence Creative Commons Attribution - Pas d'Utilisation Commerciale 4.0 International. 


\section{Péter Pomozi : Kis nyelv-nagy stratégia. Az észt nyelvpolitikai modell}

[Une petite langue, une grande stratégie. Le modèle estonien de politique linguistique], Budapest, 2011, 154 p.

\section{Eva Toulouze}

1 Le contenu de ce petit livre, édité par l'auteur avec le soutien du ministère estonien de l'Éducation et de la Recherche, est dans sa plus grande partie une traduction en hongrois de la loi estonienne sur la langue. Notons que c'est le premier volume d'une série dirigée par Péter Pomozi et András Bereczki intitulée « Petite bibliothèque sur la culture estonienne ». Ce texte est cependant pourvu de deux textes introductifs posant le contexte, aussi bien de la publication que de la loi en tant que telle.

Ce livre est un écho à l'article de Birute Klaas-Lang dans un récent numéro des Études finno-ougriennes ${ }^{1}$. C'est la raison pour laquelle il nous a semblé pertinent de le présenter en quelques mots, d'autant qu'il nous parait important de suivre avec attention les publications de nos collègues finno-ougristes dans d'autres pays, et surtout là où ces études sont particulièrement développées.

Péter Pomozi, le titre nous le montre d'emblée, est admiratif devant la politique linguistique estonienne. Il la considère même comme un modèle, sous-entendant par là que d'autres auraient toutes les raisons de s'en inspirer. Deux éléments ressortent clairement de son introduction. Tout d'abord, Pomozi a en ligne de vue son propre pays et la nécessité, en Hongrie aussi, de concevoir une politique linguistique cohérente. Il ne développe pas ce thème, qui apparait avant tout sous forme allusive. Mais il est clair que l'auteur entend faire passer des messages qui pourraient être pertinents dans son contexte propre.

4 Sur le contenu même de la politique estonienne, ce qui ressort de son introduction est qu'il en admire le caractère systématique et cohérent. Le législateur estonien et les autorités compétentes ont lancé une réflexion sur la langue (l'article de Birute KlaasLang l'illustre bien), prenant en compte tous les paramètres qui influent sur ce domaine, et ils en tirent des conséquences. Ce qui plaît à Pomozi, c'est que les Estoniens ont sur la langue une politique interventionniste. Ils ne laissent pas les choses suivre 
leur cours en relevant des tendances, ils ne sont pas neutres mais ont des objectifs qu'ils se donnent les moyens de mettre en œuvre. Il s'agit aussi bien de garantir la place de la langue estonienne dans la société, la correction de la langue utilisée, et sa diffusion auprès des résidents estoniens dont elle n'est pas la langue maternelle.

Pomozi insiste sur le travail lexicographique réalisé en Estonie et surtout sur sa continuité : on s'interroge sur l'évolution du lexique et l'on met régulièrement à jour les outils mis à disposition de la population. Clairement, l'usage d'Internet dans cette entreprise attire l'admiration de l'auteur.

6 Jüri Valge présente le contexte de mise au point de la loi : les programmes cadres, les lois précédentes, mais aussi le contexte politique, avec le passage des écoles de langue russe à un curriculum à $60 \%$ en estonien. La loi elle-même a été traduite par Pomozi, qui l'a enrichie de nombreuses notes expliquant les passages qui manquent de transparence pour un lecteur hongrois. Ce sera certainement là un petit ouvrage de référence bien utile en Hongrie pour ceux à qui la politique linguistique tient à cœur.

\section{NOTES}

1. http://efo.revues.org/515. Pour la version estonienne, http://efo.revues.org/882.

\section{INDEX}

Keywords : Language Policy

Thèmes : sociolinguistique

Index chronologique : XXIe siècle

motscleset keelepoliitika

Mots-clés : politique linguistique

Index géographique : Estonie, Hongrie

motsclesru ЯзыковАЯ ПолИТИКА 